\title{
Excessive cardiac insulin signaling exacerbates systolic dysfunction induced by pressure overload in rodents
}

\author{
Ippei Shimizu, ${ }^{1}$ Tohru Minamino,,${ }^{1,2}$ Haruhiro Toko, ${ }^{1}$ Sho Okada, ${ }^{1}$ Hiroyuki Ikeda, ${ }^{1}$ Noritaka Yasuda, ${ }^{1}$ \\ Kaoru Tateno, ${ }^{1}$ Junji Moriya, ${ }^{1}$ Masataka Yokoyama, ${ }^{1}$ Aika Nojima, ${ }^{1}$ Gou Young Koh, ${ }^{3}$ \\ Hiroshi Akazawa, ${ }^{1}$ Ichiro Shiojima, ${ }^{4}$ C. Ronald Kahn, ${ }^{5}$ E. Dale Abel, ${ }^{6}$ and Issei Komuro ${ }^{1,4}$ \\ 1Department of Cardiovascular Science and Medicine, Chiba University Graduate School of Medicine, Chiba, Japan. \\ 2PRESTO, Japan Science and Technology Agency, Saitama, Japan. ${ }^{3}$ Biomedical Research Center and Biological Sciences, \\ Korea Advanced Institute of Science and Technology, Daejeon, Korea. ${ }^{4}$ Department of Cardiovascular Medicine, Osaka University School of Medicine, \\ Osaka, Japan. ${ }^{5}$ Joslin Diabetes Center, Harvard Medical School, Boston, Massachusetts, USA. ${ }^{6}$ Division of Endocrinology, \\ Metabolism and Diabetes and Program in Molecular Medicine, University of Utah School of Medicine, Salt Lake City, Utah, USA.
}

\begin{abstract}
Although many animal studies indicate insulin has cardioprotective effects, clinical studies suggest a link between insulin resistance (hyperinsulinemia) and heart failure (HF). Here we have demonstrated that excessive cardiac insulin signaling exacerbates systolic dysfunction induced by pressure overload in rodents. Chronic pressure overload induced hepatic insulin resistance and plasma insulin level elevation. In contrast, cardiac insulin signaling was upregulated by chronic pressure overload because of mechanical stretch-induced activation of cardiomyocyte insulin receptors and upregulation of insulin receptor and Irs1 expression. Chronic pressure overload increased the mismatch between cardiomyocyte size and vascularity, thereby inducing myocardial hypoxia and cardiomyocyte death. Inhibition of hyperinsulinemia substantially improved pressure overloadinduced cardiac dysfunction, improving myocardial hypoxia and decreasing cardiomyocyte death. Likewise, the cardiomyocyte-specific reduction of insulin receptor expression prevented cardiac ischemia and hypertrophy and attenuated systolic dysfunction due to pressure overload. Conversely, treatment of type 1 diabetic mice with insulin improved hyperglycemia during pressure overload, but increased myocardial ischemia and cardiomyocyte death, thereby inducing HF. Promoting angiogenesis restored the cardiac dysfunction induced by insulin treatment. We therefore suggest that the use of insulin to control hyperglycemia could be harmful in the setting of pressure overload and that modulation of insulin signaling is crucial for the treatment of $\mathrm{HF}$.
\end{abstract}

\section{Introduction}

Cardiac hypertrophy is defined as an increment of ventricular mass resulting from increased cardiomyocyte size and is the adaptive response of the heart to an increased hemodynamic load due to either physiological factors such as exercise or pathological states such as hypertension and valvular diseases (1). Exercise-induced cardiac hypertrophy does not progress to heart failure $(\mathrm{HF})(2,3)$ and therefore is thought to be "physiological hypertrophy." On the other hand, pressure overload initially induces "adaptive hypertrophy," but causes "maladaptive (pathological) hypertrophy" in the chronic phase that results in HF (1).

Several signaling pathways have been implicated in the development of physiological or pathological cardiac hypertrophy. The insulin/PI3K/Akt axis plays a crucial role in the development of physiological hypertrophy as well as in normal cardiac growth, whereas activation of G-protein-coupled receptors in collaboration with PKC and calcineurin/nuclear factor of activated T cells (NFAT) pathways is involved in the development of pathological hypertrophy (4). Although homozygous cardiomyocyte-specific insulin receptor knockout (CIRKO) mice have smaller hearts than WT controls (5), both WT and CIRKO mice have shown a compa-

Authorship note: Ippei Shimizu and Tohru Minamino contributed equally to this work.

Conflict of interest: The authors have declared that no conflict of interest exists. Citation for this article: J Clin Invest. 2010;120(5):1506-1514. doi:10.1172/JCI40096 rable increase of cardiac mass in response to pathological hypertrophic stimuli such as pressure overload (6). Overexpression of constitutively active $\mathrm{p} 110 \alpha$, a catalytic component of PI3K, in the heart has led to enhanced cardiac growth with preserved systolic function (7). Conversely, myocardial expression of dominant-negative $\mathrm{p} 110 \alpha$ has inhibited the physiological hypertrophic response during postnatal growth and following exercise in mice, whereas the response to pressure overload has not been altered (8). Likewise, homozygous Akt1-deficient mice have shown defective exercise-induced cardiac hypertrophy (9), further supporting a crucial role of the insulin/PI3K/Akt pathway in physiological hypertrophy and growth of the heart.

Besides their role in physiological hypertrophy and normal cardiac growth, insulin signals may induce pathological hypertrophic responses. It has been shown that chronic hyperinsulinemia stimulates angiotensin II signaling that is involved in pathological hypertrophy (10). Mild to moderate activation of Akt was shown to induce cardiac hypertrophy with preservation of function $(11,12)$, whereas high levels of activated Akt expression in the heart led to pathological hypertrophy (13). Short-term Akt activation induced physiological cardiac hypertrophy, but constitutive activation of this pathway led to cardiac dysfunction (14). In this state, coordinated tissue growth and angiogenesis in the heart were disrupted, leading to myocardial hypoxia (14). Likewise, it has been demonstrated that chronic pressure overload increases the mismatch between cardiomyocyte size and vascularity and therefore induces 

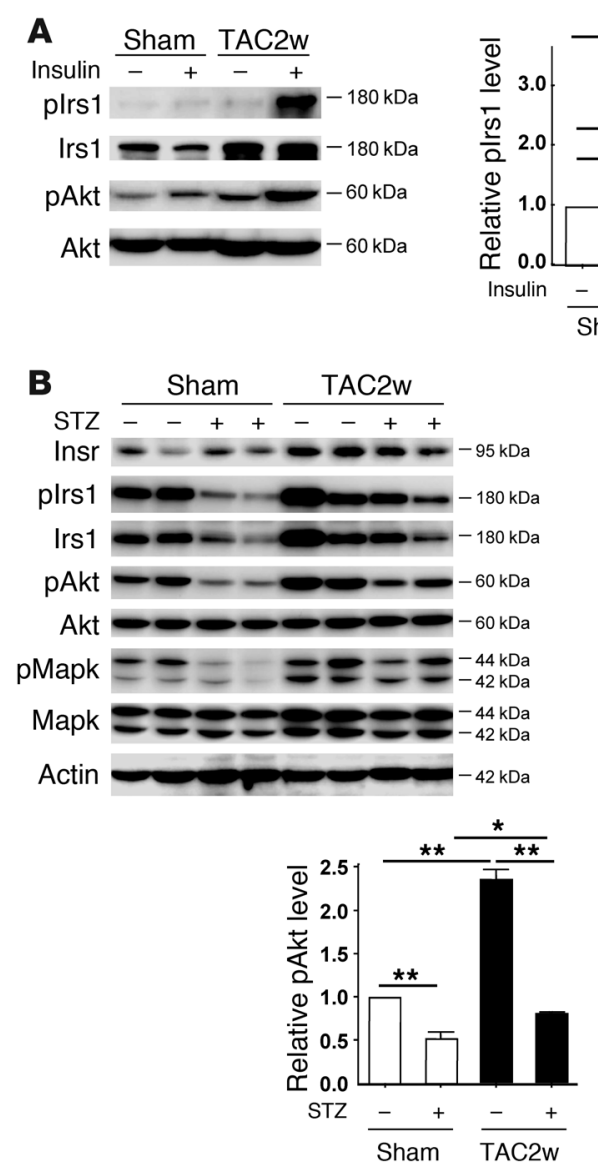
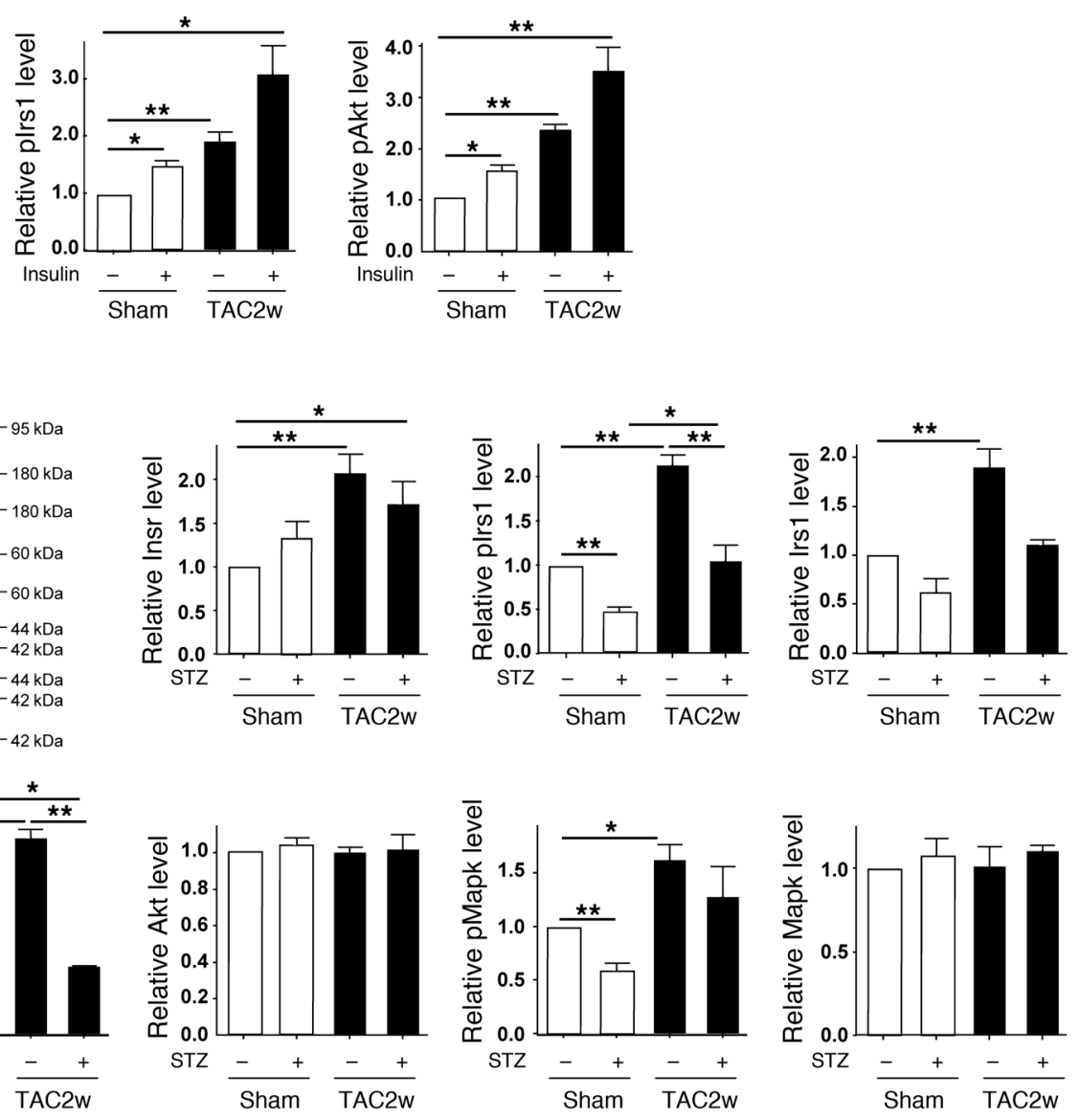

Figure 1

Upregulation of cardiac insulin signals by pressure overload. (A) Mice were subjected to TAC or sham operation (sham), and heart samples were obtained 2 weeks later. Mice were starved for 6 hours, and insulin or PBS was injected before sacrifice. plrs 1 and pAkt levels in the heart were examined by Western blot analysis. The graphs indicate relative expression levels of plrs 1 and pAkt. $n=3$. TAC2w, 2 weeks after TAC. (B) Mice were subjected to TAC or sham operation and were sacrificed 2 weeks later. Components of the insulin signaling pathway in the heart were examined by Western blot analysis. The graphs indicate relative expression levels of these signaling molecules. $n=3$. Data are shown as mean \pm SEM. ${ }^{\star} P<0.05 ;{ }^{* *} P<0.01$.

myocardial hypoxia and cardiomyocyte death, leading to cardiac dysfunction (15). Moreover, intensive glycemic control of diabetic patients by insulin treatment has been reported to increase cardiovascular events (16). In the present study, we examined the role of insulin signaling in the development of cardiac dysfunction induced by pressure overload.

\section{Results}

Cardiac insulin signaling is activated by pressure overload. To investigate the role of the insulin signal pathway in failing hearts, we created severe transverse aortic constriction (TAC) in mice at 11 weeks of age. In this model, cardiac hypertrophy gradually progressed and reached a peak on day 7 after TAC (Supplemental Figure 1; supplemental material available online with this article; doi:10.1172/ JCI40096DS1). Systolic function was preserved until day 7 but was significantly decreased on day 14 along with left ventricular dilatation (Supplemental Figure 1). Seven and fourteen days after TAC, we treated the mice with insulin $(1 \mathrm{IU} / \mathrm{kg})$ before sacrifice and examined the downstream signaling pathway of the insulin receptor (Insr) in the heart. Insulin-induced phosphorylation of insulin receptor substrate-1 (pIrs1) and Akt (pAkt) was markedly upregulated in the hearts of the TAC group compared with the sham-operated group (Figure 1A and Supplemental Figure 2A). We also found that the insulin signal pathway was constitutively activated in the TAC hearts under fasting conditions (Figure 1B and Supplemental Figure 2B). Expression of Insr and Irs1 protein as well as pIrs1 and pAkt protein was significantly increased in the TAC heart. These results suggest that chronic pressure overload upregulates cardiac insulin signaling. Enhanced insulin signaling was also observed in the hearts of spontaneously hypertensive rats (Supplemental Figure 3A).

Reduction of plasma insulin ameliorates systolic dysfunction induced by pressure overload. To determine whether upregulation of cardiac insulin signals has a pathological role in HF, we treated the mice with streptozotocin (STZ) $(50 \mathrm{mg} / \mathrm{kg}$ i.p. for 5 days) at 4 weeks before TAC. Injection of STZ markedly decreased plasma insulin to below detectable levels, while the plasma glucose level gradually increased (Supplemental Figure 4). Pressure overload led to prominent cardiac hypertrophy along with upregulation of cardiac insulin signaling (Figure 1B and Figure 2, A and B). Systolic function was impaired and the left ventricular systolic dimension 

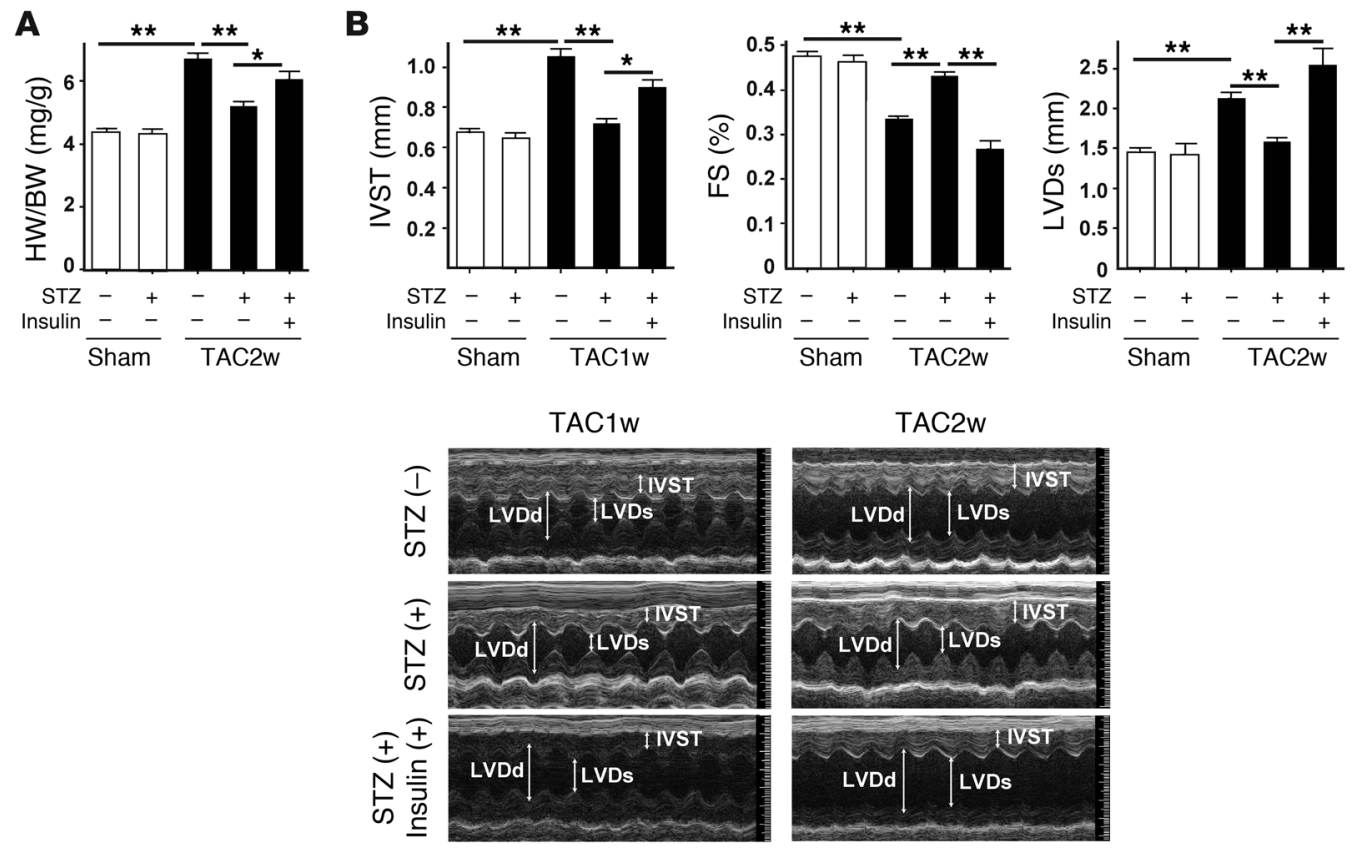

\section{Figure 2}

Depletion of plasma insulin attenuates systolic dysfunction induced by pressure overload. (A) STZ- or vehicle-treated mice were subjected to TAC or sham operation. The heart weight/body weight (HW/BW) ratio was measured 2 weeks after operation. In the insulin-treated group, daily i.p. injection of insulin $(0.1 \mathrm{lU} / \mathrm{g} / \mathrm{d}$ ) was performed from 9 weeks (2 weeks after STZ treatment) to 13 weeks of age (2 weeks after TAC). $n=22-24$. (B) Cardiac hypertrophy and systolic function of the animals prepared for A were estimated by echocardiography at 1 week (IVST) or 2 weeks (FS and LVDs) after operation. Photographs show representative results of echocardiography (M-mode). $n=6-10$. Data are shown as mean \pm SEM. ${ }^{\star} P<0.05 ;{ }^{* \star} P<0.01$. IVST, intraventricular septal thickness; FS, fractional shortening.

(LVDs) was increased at 14 days after TAC (Figure 2, A and B). These alterations were significantly ameliorated in the mice treated with STZ (Figure 1B and Figure 2, A and B). Similar results were obtained at 6 weeks after TAC (Supplemental Figure 2C). We next examined the effect of insulin on cardiac function in this setting. STZ-treated mice were subjected to daily injection of insulin $(0.1$ $\mathrm{IU} / \mathrm{g} / \mathrm{d}$ from 9 weeks to 13 weeks of age) and to TAC at 11 weeks of age. Insulin treatment significantly improved hyperglycemia (Supplemental Figure 4). However, this treatment significantly enhanced cardiac hypertrophy and decreased systolic function along with left ventricular dilatation (Figure 2, A and B), indicating that insulin signaling influenced the development of systolic dysfunction due to pressure overload.

Reduction of plasma insulin inbibits cardiac bypoxia during pressure overload. We have recently demonstrated that cardiac angiogenesis is critically involved in the adaptive mechanism of cardiac hypertrophy and that an increased mismatch between cardiomyocyte size and vascularity is a crucial determinant of the transition from cardiac hypertrophy to HF (15). Consistent with our previous results, chronic pressure overload increased the cross-sectional area (CSA) of cardiomyocytes and decreased the relative vascularity (number of vessels/number of cardiomyocytes/CSA) (Figure 3, A-C), which in turn led to exacerbation of myocardial hypoxia (Figure 3D) and cardiomyocyte death (Figure 3E). In contrast, the increase of CSA after TAC was significantly attenuated by STZ treatment and the relative vascular density was markedly increased (Figure $3, \mathrm{~A}-\mathrm{C}$ ). Consequently, depletion of plasma insulin prevented cardiac hypoxia and cardiomyocyte death during chronic pres- sure overload (Figure 3, D and E). Conversely, insulin treatment of STZ-treated mice increased CSA and decreased relative vascular density, thereby exacerbating cardiac hypoxia and cardiomyocyte death (Figure 3, A-E). Additional treatment with the proangiogenic factor cartilage oligomeric matrix protein-angiopoietin-1 (COMP-Ang1) (17) increased relative vascular density and thereby improved cardiac hypoxia and systolic dysfunction (Supplemental Figure 5, A-C). We also found that a decrease of relative vascular density was associated with cardiac dysfunction, along with upregulation of insulin signaling in spontaneously hypertensive rats (Supplemental Figure 3, A-G), suggesting that cardiac insulin signaling plays a pathological role in HF by increasing a mismatch between cardiomyocyte size and vascularity.

Cardiomyocyte-specific reduction of Insr expression attenuates systolic dysfunction due to pressure overload. To further investigate the role of cardiac insulin signaling, we generated CIRKO mice by using the $\mathrm{Cre}-\mathrm{loxP}$ system. We prepared transgenic mice in which a transgene encoding Cre recombinase was driven by the cardiomyocyte-specific $\alpha$-myosin heavy chain (MHC) promoter (18). We then crossed these MHC-Cre mice with mice bearing floxed Insr alleles (19) and produced TAC in the resulting mice. Since homozygous CIRKO $\left(\right.$ Ins fllox/flox $^{-\mathrm{Cre}^{+}}$) mice have been shown to develop systolic dysfunction in response to pressure overload (6), we utilized heterozygous CIRKO (Insr flox/+ $\mathrm{Cre}^{+}$) mice with reduced cardiac expression of Insr (Figure 4A). These mice had a normal heart size and normal systolic function under physiological conditions (Figure 4, B and C). However, cardiac insulin signaling was markedly attenuated in the TAC heart of CIRKO mice (Figure 4B), and therefore chronic pressure 
A
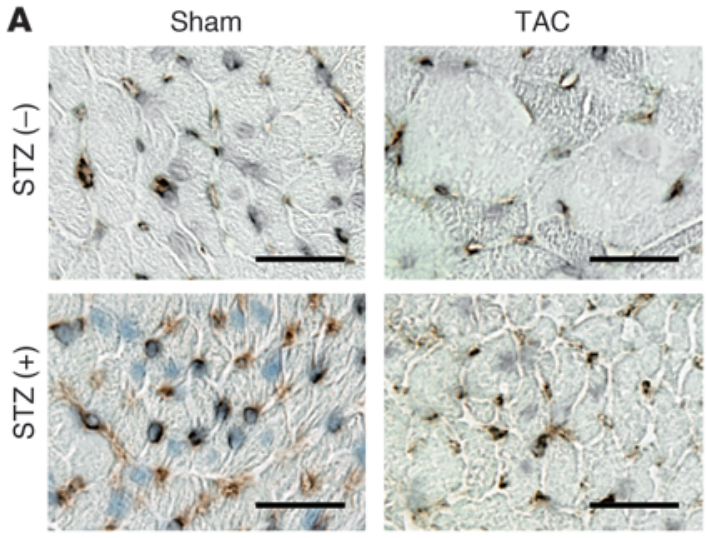

声
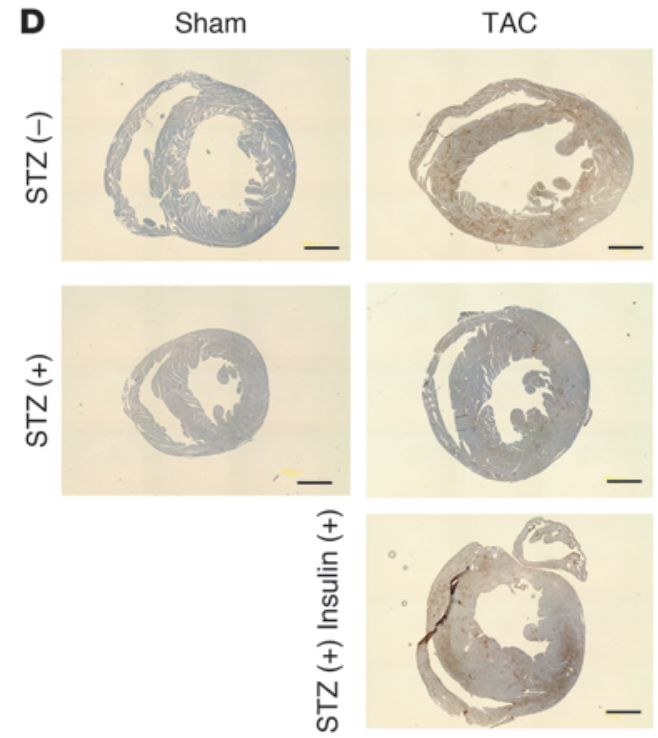

B

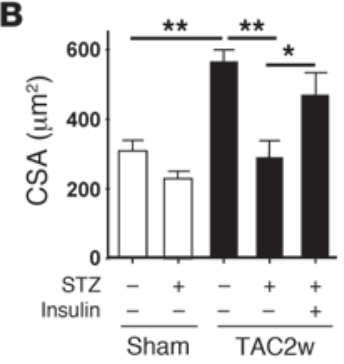

C

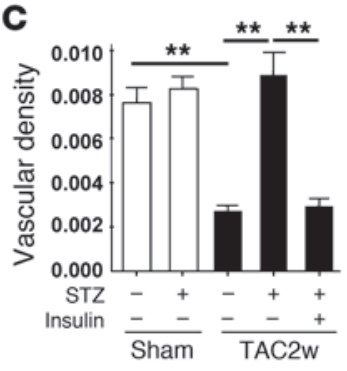

E

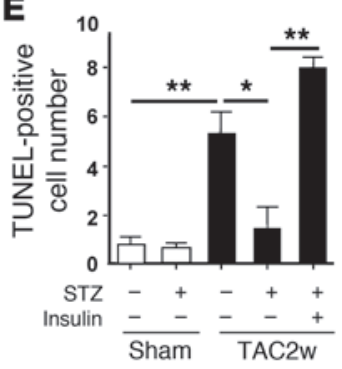

\section{Figure 3}

Reduction of plasma insulin inhibits cardiac hypoxia due to pressure overload. (A) Animals were prepared as described for Figure 2A. Immunohistochemistry using antibodies against platelet and endothelial cell adhesion molecule (dark brown) and dystrophin (light brown) was performed at 2 weeks after operation. Scale bars: $20 \mu \mathrm{m}$. (B and C) CSA of cardiomyocytes (B) and relative vascular density (C) were estimated as described in Methods. $n=4-5$. (D) Cardiac ischemia (brown) in mice prepared as described for Figure 2A was estimated with a Hypoxyprobe-1. Scale bars: $1 \mathrm{~mm}$. (E) Number of TUNEL-positive cells per $1 \times 10^{4}$ cardiomyocytes. $n=4-6$. Data are shown as mean \pm SEM. ${ }^{*} P<0.05 ;{ }^{*} P<0.01$. overload caused less severe hypertrophy than in WT mice (Figure 4, $\mathrm{C}$ and D). Both systolic dysfunction and left ventricular dilatation were significantly inhibited in CIRKO mice compared with their littermate controls (Figure 4D and Supplemental Figure 6). Histological examination showed that the increase of CSA after TAC was significantly attenuated and relative vascular density was markedly increased in CIRKO mice (Figure 5, A-C). In consequence, the number of dead cardiomyocytes was significantly smaller in CIRKO mice than in their littermate controls (Figure 5D).

To investigate the role of Akt in HF induced by pressure overload, we utilized heterozygous Akt1-deficient $\left(A k t 1^{+/-}\right)$mice. Two weeks after TAC operation, both systolic dysfunction and left ventricular dilatation were significantly inhibited in $A k t 1^{+/-}$mice compared with their littermate controls (Figure 6A). Histological examination showed that the increase of CSA after TAC was significantly attenuated and relative vascular density was markedly increased in $\mathrm{Akt1}^{+/-}$mice (Figure 6, B and C), which was associated with decreased activation of Akt (Figure 6D). These data suggest that sustained activation of Akt could cause cardiac dysfunction under chronic pressure overload.

Mechanism of enhanced cardiac insulin signaling due to pressure overload. To investigate the additional mechanisms by which chronic pressure overload enhances insulin signaling in the heart, we examined pIrs1 levels immediately after TAC. Western blot analysis revealed that pressure overload markedly increased the pIrs 1 level from as early as 1 minute after the operation (Figure 7A). Such activation was significantly attenuated in both heterozygous and homozygous CIRKO mice (Figure 7A and Supplemental Figure 7), suggesting that mechanical stress may also upregulate the insulin signaling pathway via direct activation of Insr independent of its ligand. To further investigate the influence of mechanical stress on insulin signaling, we stretched cultured cardiomyocytes by $20 \%$ and examined the changes in the pIrs1 level. Consistent with our hypothesis, stretching of cardiomyocytes led to marked activation of insulin sig- 
A

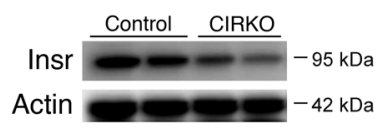

B

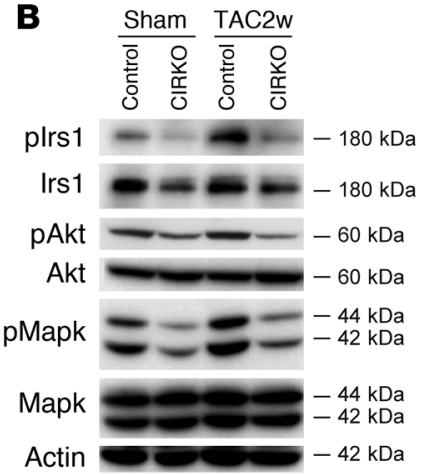

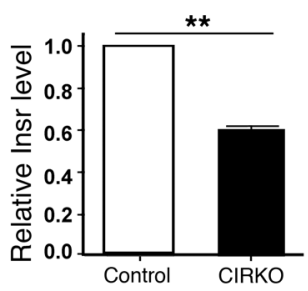
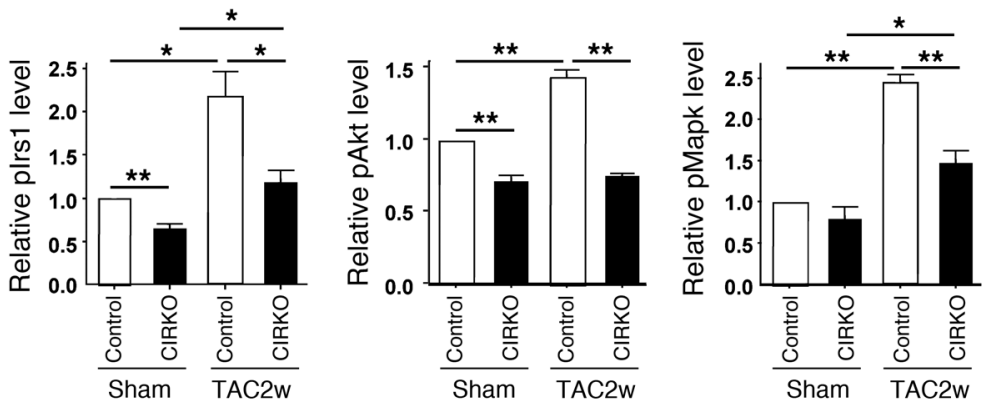

D
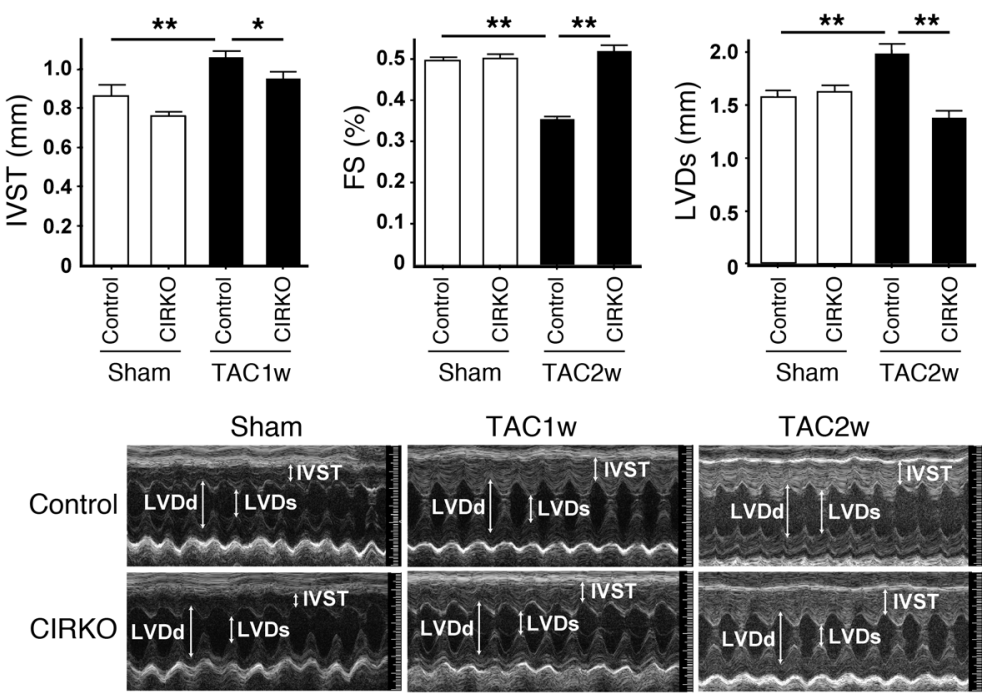

TAC1w

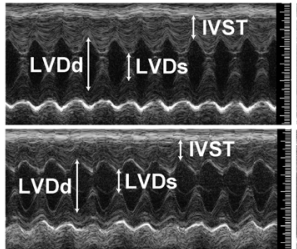

TAC2W

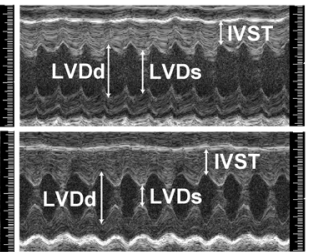

Figure 4

Cardiomyocyte-specific reduction of Insr expression attenuates systolic dysfunction due to pressure overload. (A) Western blot analysis of Insr expression in the hearts of CIRKO mice $\left(I n s r^{f l o x /+} \mathrm{Cre}^{+}\right)$and their littermate controls (control). Graphs indicate relative expression levels of Insr. $n=3$. (B) CIRKO mice $\left(I n s r^{f l o x} /+\mathrm{Cre}^{+}\right)$or littermate controls were subjected to TAC or sham operation, and components of the insulin signaling pathway in the heart were examined by Western blot analysis at 2 weeks after operation. Graphs indicate relative expression levels of these signaling molecules. $n=3$. (C) The heart weight/body weight ratio of animals prepared as described in A was measured at 2 weeks after operation. $n=7-9$. (D) Cardiac hypertrophy and systolic function of animals prepared as described in A were assessed by echocardiography at 1 week (IVST) or 2 weeks (FS and LVDs) after operation. Photographs show representative results of echocardiography (M-mode). $n=8-13$. Data are shown as mean \pm SEM. ${ }^{\star} P<0.05 ;{ }^{*} P<0.01$.

naling (Figure 7B). This activation was abolished by knockdown of Insr expression (Figure 7C), whereas knockdown of Igf1 or the Igf1 receptor showed a marginal effect (Supplemental Figure 8). These results suggest that mechanical stress mainly enhances insulin signaling through Insr and that Igf1 and the Igf1 receptor contribute to stretch-induced activation of this signaling to a lesser extent. This is similar to the known direct activation of the angiotensin II type I receptor by mechanical stress, which contributes to pathological hypertrophy (20); however, the precise mechanism of how mechanical stress activates insulin signaling needs further investigation.
There is accumulating evidence that suggests a potential relationship between insulin resistance and cardiac hypertrophy $(21,22)$. Therefore we examined plasma glucose and insulin levels in mice subjected to chronic pressure overload. Both glucose and insulin levels were significantly higher in the TAC group than in the shamoperated group (Figure 7D). More importantly, the homeostasis model assessment-insulin resistance (HOMA-IR) index was markedly elevated in the TAC group (Figure 7D). Furthermore, insulininduced phosphorylation of Akt was impaired in the liver of the TAC group compared with the sham-operated group (Figure 7E). 
A
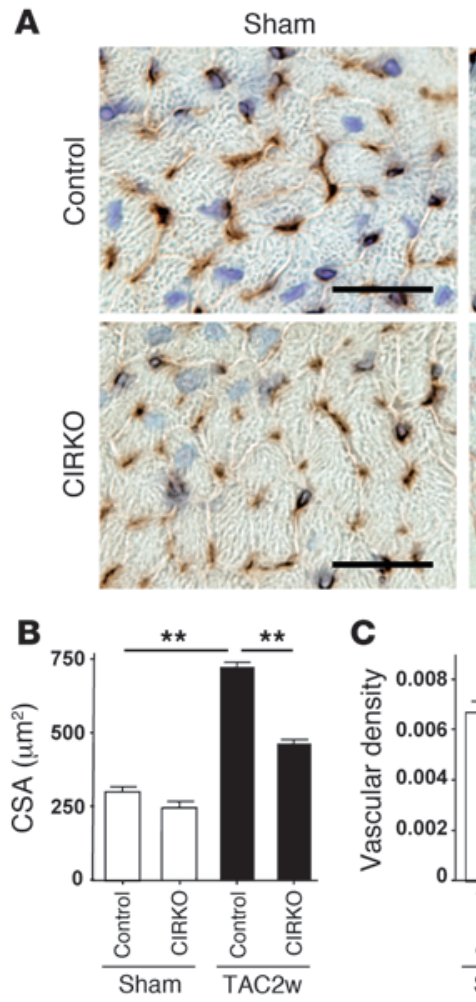

C

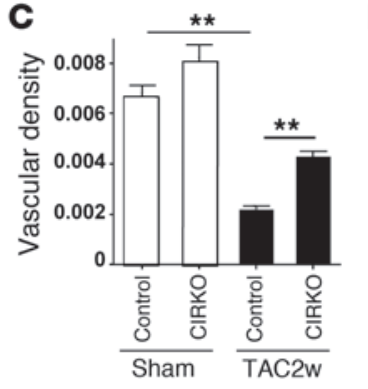

TAC2w

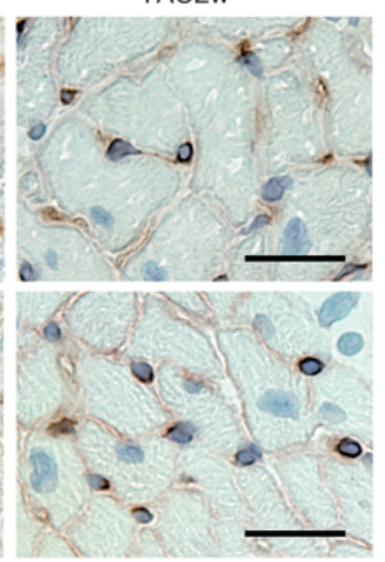

D

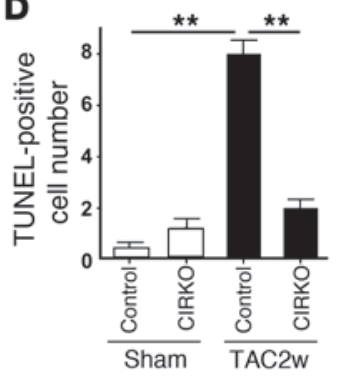

\section{Figure 5}

Cardiomyocyte-specific reduction of Insr expression attenuates cardiac hypoxia due to pressure overload. (A) CIRKO mice (Insr flox/+Cre+) or littermate controls were subjected to TAC or sham operation. Immunohistochemistry using antibodies against platelet and endothelial cell adhesion molecules (dark brown) and dystrophin (light brown) was performed at 2 weeks after operation. Scale bars: $20 \mu \mathrm{m}$. (B and C) CSA of cardiomyocytes (B) and relative vascular density (C) were estimated as described in Methods. $n=4-5$. (D) Number of TUNEL-positive cells per $1 \times 10^{4}$ cardiomyocytes. $n=4-5$. Data are shown as mean \pm SEM. ${ }^{\star} P<0.05 ;{ }^{* \star} P<0.01$.
These results suggest that chronic pressure overload induces hepatic insulin resistance, thereby inducing hyperinsulinemia, whereas there is no cardiac insulin resistance due to direct activation of Insr as well as to upregulation of Insr and Irs1.

\section{Discussion}

A number of clinical studies have strongly indicated the link between insulin resistance and nonischemic HF (23-26). Approximately two-thirds of patients with essential hypertension have abnormal glucose metabolism (27), and there is a positive relationship between cardiac hypertrophy and the plasma insulin concentration (28), suggesting that elevation of insulin contributes to myocardial growth in the presence of chronic pressure overload. Consistent with these reports, we found that chronic pressure overload induced hepatic insulin resistance and increased the plasma insulin level. Myocardial stretch activated Insr, and chronic pressure overload not only increased the activity of insulin signaling (pIrs1 and pAkt levels), but also upregulated the expression of Insr and Irs 1 protein. This in turn facilitated activation of cardiac insulin signals by hyperinsulinemia. Such activation enhanced the mismatch between vascularity and cardiomyocyte size and increased cardiomyocyte death. This increase was associated with systolic dysfunction and may be one of the causes of HF induced by chronic pressure overload. However, we have not excluded other mechanisms by which excessive insulin signals promote cardiac dysfunction during pressure overload. For example, cardiac hypoxia may affect metabolism and contraction of myocytes with their viability being unchanged. Indeed, we only showed evidence for tissue hypoxia in the TAC heart by using pimonidazole, which may not be sufficient. We have not demonstrated that inhibition of cardiomyocyte death attenuates systolic dysfunction of the TAC heart. Accordingly, we cannot definitively conclude that hypoxia- induced cardiomyocyte death was essential for the development of HF. It has been reported that endothelial cells in the heart release a variety of factors, such as neuregulin and nitric oxide, that regulate survival and function of cardiomyocytes and that endothelialmyocardial interaction plays a crucial role in maintaining systolic function (29). Thus, it is also possible that a decrease of relative vascular density in the TAC heart impairs such paracrine mechanisms, leading to systolic dysfunction.

Our results were similar to those of the study with conditional Akt transgenic animals (14). In this model, Akt signaling could be switched on or off in the heart. These mice developed physiological hypertrophy following short-term induction, but exhibited pathological hypertrophy with longer periods of Akt activation due to an imbalance between cardiac growth and angiogenesis. Interestingly, cardiac dysfunction was further impaired when Akt was switched off after prolonged activation. These results suggest that Akt signaling itself is beneficial for maintenance of systolic function in this model; however, excessive cardiac growth with insufficient angiogenesis causes pathological hypertrophy. Thus, although insulin/Akt signaling has been implicated in the development of physiological hypertrophy, constitutive activation of these signals can induce HF when coordinated tissue growth and angiogenesis are disrupted.

Alterations of myocardial substrate metabolism have been implicated in the pathogenesis of contractile dysfunction and HF $(21$, 30). Studies on animal models of HF have demonstrated that, during transition from cardiac hypertrophy to ventricular dysfunction, expression of genes encoding for mitochondrial fatty acid (FA) $\beta$-oxidation enzymes shows a coordinated decrease, resulting in a shift of myocardial metabolism that recapitulates the fetal heart gene program, with glucose instead of FA becoming the primary energy substrate $(31,32)$. Clinical studies have revealed that patients with nonischemic cardiomyopathy exhibit alterations of 
A
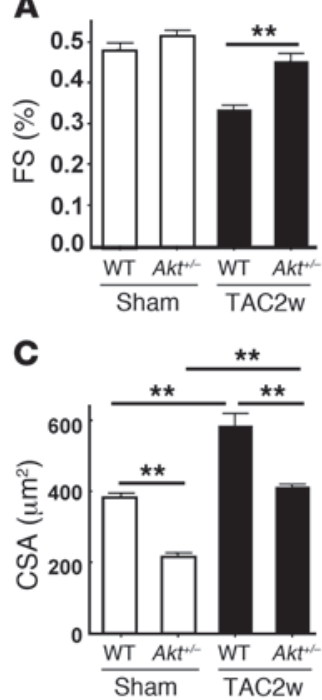

D

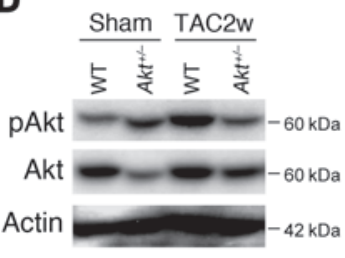

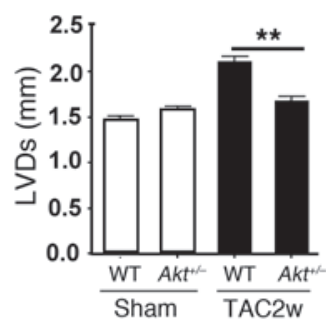
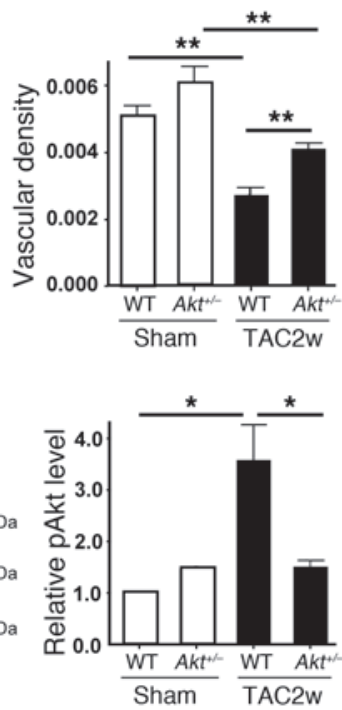

B
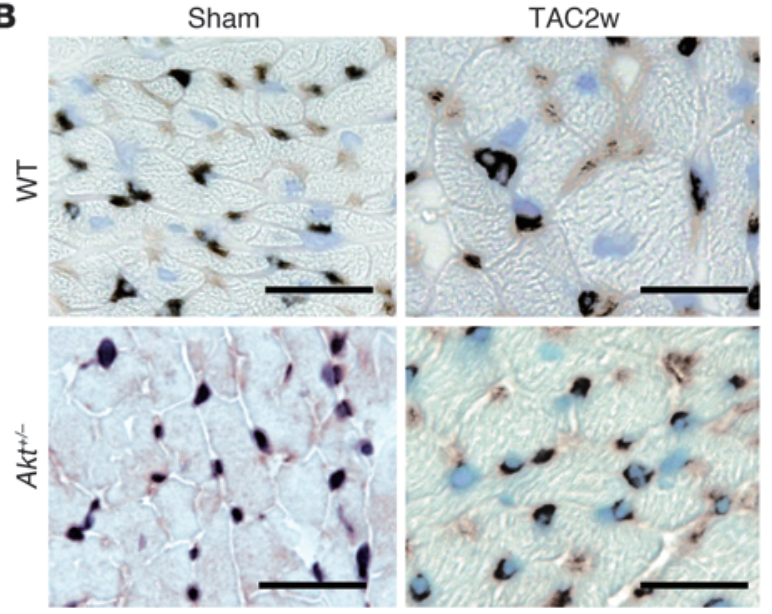

Figure 6

Reduced activation of Akt attenuates systolic dysfunction due to pressure overload. (A) Akt1-deficient (Akt1+l-) mice and WT littermates were subjected to TAC or sham operation. Cardiac hypertrophy and systolic function were assessed by echocardiography at 2 weeks after operation. $n=4-6$. (B) Immunohistochemistry using antibodies against platelet and endothelial cell adhesion molecules (dark brown) and dystrophin (light brown) was performed at 2 weeks after operation. Scale bars: $20 \mu \mathrm{m}$. (C) CSA of cardiomyocytes and relative vascular density were estimated as described in Methods. $n=3$. (D) pAkt and Akt levels in the heart at 2 weeks after operation were examined by Western blot analysis. Graphs indicate relative expression levels of pAkt and Akt. $n=3$. Data are shown as mean \pm SEM. ${ }^{*} P<0.05 ;{ }^{* \star} P<0.01$.

myocardial metabolism that are characterized by a decrease of FA metabolism and an increase of myocardial glucose metabolism, a pattern similar to that shown in animal models of HF (33). Under these conditions, increased FA metabolism in the heart is pathogenic and the extent of abnormal FA metabolism predicts both morphologic changes of the heart and a poor clinical outcome (34). In this respect, activation of the insulin/Akt pathway in the failing heart appears to be an adaptive response, but constitutive activation of this pathway also leads to activation of growth signals that results in dysregulated hypertrophy, cardiac hypoxia, and systolic dysfunction. Thus, a metabolic modulator that increases glucose uptake (or decreases FA metabolism) without activation of insulin signaling would be a better strategy for the treatment of HF because these patients have systemic insulin resistance. Our results also suggest that the use of insulin to control hyperglycemia can be harmful, especially in the setting of pressure overload, a finding that is consistent with the outcome of a recent clinical trial (16).

Multiple counterregulatory hormones and cytokines are upregulated in HF and are likely to play a role in insulin resistance and altered glucose disposition (21). Upregulation of catecholamines not only contributes directly to the pathogenesis of cardiomyopathy but also increases insulin resistance and thereby indirectly affects systolic function. We also found that chronic pressure overload increased the production of proinflammatory cytokines by adipose tissue, thus promoting systemic insulin resistance (I. Shimizu and T. Minamino, unpublished observations). Further investigation of the link between insulin resistance and HF will continue to provide novel insights into the treatment of HF.

\section{Methods}

Animal models. All animal study protocols were approved by the Chiba University Review Board. C57BL/6 mice were purchased from SLC Japan. TAC was performed as described previously (15) in 11-week-old male mice. Sham-operated mice underwent the same procedure except for aortic constriction. For the type 1 diabetic model, 7-week-old male C57BL/6 mice were treated with i.p. STZ in $0.1 \mathrm{M}$ sodium citrate ( $\mathrm{pH} 4.5$ ) at a dose of $50 \mathrm{mg} / \mathrm{kg}$ for 5 days. TAC was performed 4 weeks after STZ treatment. In the insulin-treated group, mice received daily i.p. injection of insulin $(0.1 \mathrm{IU} / \mathrm{g} / \mathrm{d})$ from 9 weeks ( 2 weeks after STZ treatment) to 13 weeks of age ( 2 weeks after TAC). In some experiments, mice received an i.p. injection of insulin $(1 \mathrm{IU} / \mathrm{kg}) 30$ minutes before sacrifice to investigate the insulin sensitivity of various organs. Akt1-deficient mice $\left(A k t 1^{+/}\right)$were a gift from Morris J. Birnbaum (University of Pennsylvania School of Medicine, Philadelphia, Pennsylvania, USA). The generation and genotyping of Akt1-deficient mice, floxed Insr mice, and MHC-Cre mice have been described previously (18, 19, 35). Littermate controls have the genotype Insrflox/+ or Insr flox/flox. We 
A

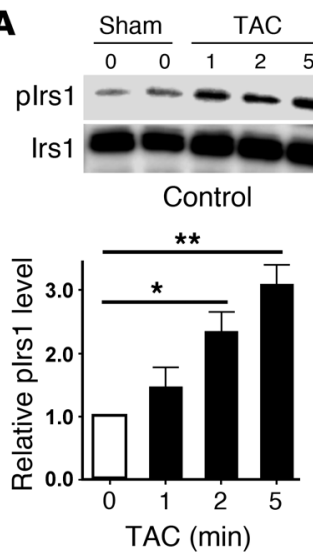

Sham $\frac{\text { TAC }}{0}$
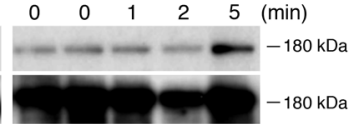

CIRKO (Insriloxtlox $\left.\mathrm{Cre}^{+}\right)$

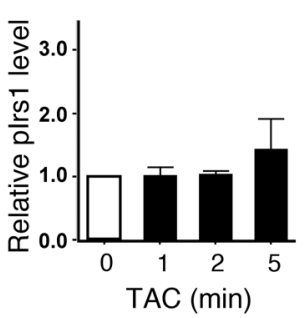

B
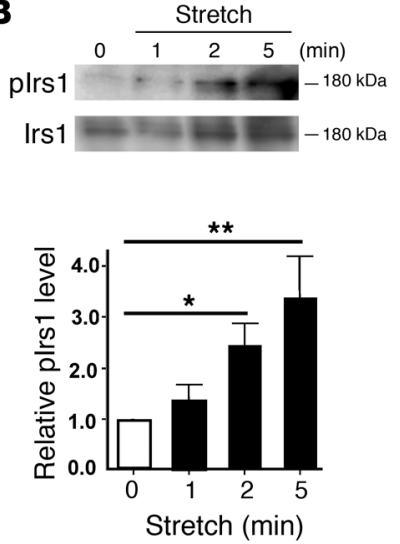

C

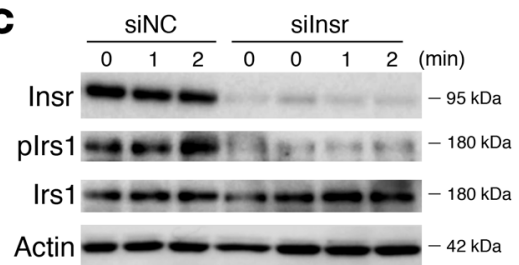

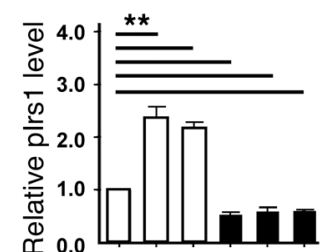

Stretch (min) $\begin{array}{lll}0 \quad 1 \quad 2 & 0 \quad 1 \quad 2\end{array}$
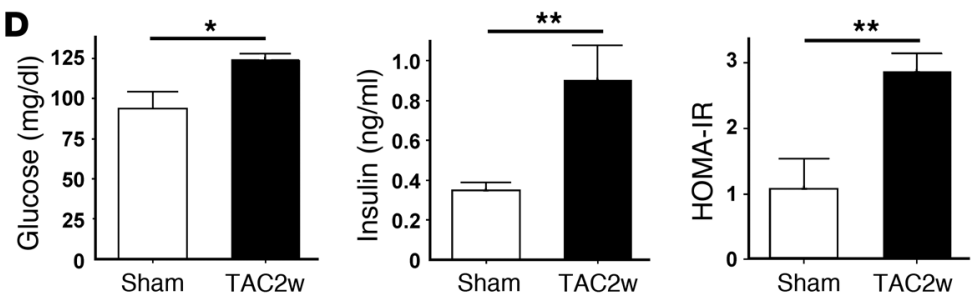

$\mathbf{E}$
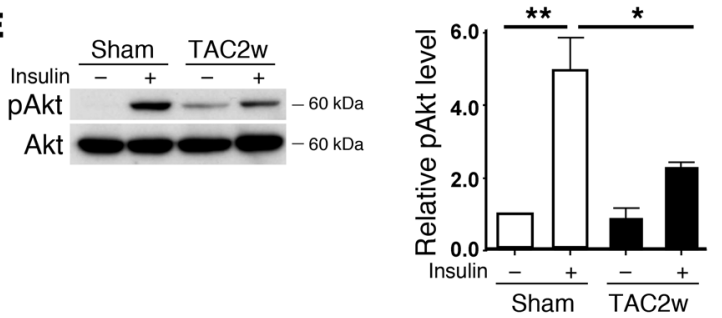

administered adenoviral vector encoding COMP-Ang1 to mice i.v. after TAC operation as previously described (17).

Physiological and histological analyses. Echocardiography was performed with a Vevo 770 High Resolution Imaging System (Visual Sonics Inc.). To minimize variation of the data, the heart rate was always approximately $500-600$ beats per minute when cardiac function was assessed. Cardiac tissue was fixed by perfusion with $4 \%$ paraformaldehyde. The fixed sample was immersed in OCT compounds (Miles Inc.) and snap-frozen in liquid nitrogen to prepare cryostat sections. Frozen cross sections of hearts were immunohistochemically double stained with antibodies for PECAM (BD Biosciences - Pharmingen) and dystrophin (Novocastra Laboratories). For measurement of the CSA of cardiomyocytes, 50 randomly selected cardiomyocytes in a left ventricular cross section were measured by tracing dystrophin immunostaining with NIH ImageJ software (http://rsbweb. nih.gov/ij/). Using the same sections, the number of PECAM-positive vessels was counted, and vascular density was estimated as the number of microvessels/number of cardiomyocytes/CSA. Tissue hypoxia was esti- mated with the Hypoxyprobe-1 (Chemicon) according to the manufacturer's instructions. Briefly, an i.p. injection of pimonidazole $(60 \mathrm{mg} / \mathrm{kg})$ was performed 90 minutes before sacrifice. Heart samples were harvested and fixed in $10 \%$ formalin overnight. The samples were embedded in paraffin, sectioned at $4-\mu \mathrm{m}$ thickness, and stained with the Hypoxyprobe-1 monoclonal antibody (clone 4.3.11.3), which binds to protein adducts of pimonidazole in hypoxic cells. TUNEL labeling was performed according to the manufacturer's protocol (In Situ Apoptosis Detection Kit; TaKaRa) in combination with immunostaining for appropriate cell markers.

Western blot analysis. Whole-cell lysates were prepared in lysis buffer $(10 \mathrm{mM}$ Tris-HCl, pH 8, 140 mM NaCl, 5 mM EDTA, 0.025\% NaN3, 1\% Triton X-100, $1 \%$ deoxycholate, $0.1 \%$ SDS, $1 \mathrm{mM}$ PMSF, $5 \mu \mathrm{g} / \mathrm{ml}$ leupeptin, $2 \mu \mathrm{g} / \mathrm{ml}$ aprotinin, $50 \mathrm{mM} \mathrm{NaF}$, and $1 \mathrm{mM} \mathrm{Na}_{2} \mathrm{VO}_{3}$ ). The lysates (40-50 $\mu \mathrm{g}$ ) were resolved by SDS-PAGE. Proteins were transferred to a PVDF membrane (Millipore), which was incubated with the primary antibody followed by anti-rabbit or anti-mouse immunoglobulin-G conjugated with horseradish peroxidase (Jackson). Specific proteins were detected by enhanced chemiluminescence 
(Amersham). The primary antibodies used for Western blotting were as follows: anti-pIrs1 antibody (Tyr612, Biomol), anti-Irs1 antibody (C20), antiAkt1 antibody (C20), anti-Insr $\beta$ antibody (C-19) (Santa Cruz Biotechnology Inc.), anti-phospho-Akt antibody (Ser473), anti-phospho-p44/42 MAP kinase antibody (Thr202/Tyr204), anti-MAP kinase (ERK1+ERK2) antibody (Invitrogen), and anti-actin antibody (Sigma-Aldrich). Plasma insulin levels were evaluated with an ELISA kit (Morinaga Institute of Biological Science Inc.) according to the manufacturer's instructions.

Cell culture. Neonatal Wistar rats were purchased from Takasugi Experimental Animal Supply. Cardiomyocytes were prepared from neonatal rats and cultured as described previously (15). Passive stretching of cultured cells was done as described previously. Cells were plated on collagen-coated silicone rubber dishes (STREX Mechanical Cell Strain Instruments), and the silicone dishes were stretched by attaching both ends of each dish firmly to a fixed frame, resulting in longitudinal stretch by $20 \%$ of the original length. siRNA targeting Insr, IGF, and the IGF-1 receptor was purchased from Invitrogen and introduced into rat cardiomyocytes by using Lipofectamine RNAiMax (Invitrogen) according to the manufacturer's instructions.

Statistics. Data are shown as the mean \pm SEM. Differences between groups were examined by 2 -tailed Student's $t$ test or ANOVA, followed by Bonferroni's correction for comparison of means. For all analyses, $P<0.05$ was considered statistically significant.

1. Frey N, Olson EN. Cardiac hypertrophy: the good, the bad, and the ugly. Annu Rev Physiol. 2003;65:45-79.

2. Adams TD, Yanowitz FG, Fisher AG, Ridges JD, Lovell K, Pryor TA. Noninvasive evaluation of exercise training in college-age men. Circulation. 1981; 64(5):958-965.

3. Pelliccia A, Maron BJ. Outer limits of the athlete's heart, the effect of gender, and relevance to the differential diagnosis with primary cardiac diseases. Cardiol Clin. 1997;15(3):381-396.

4. Heineke J, Molkentin JD. Regulation of cardiac hypertrophy by intracellular signalling pathways. Nat Rev Mol Cell Biol. 2006;7(8):589-600.

5 . Belke DD, et al. Insulin signaling coordinately regulates cardiac size, metabolism, and contractile protein isoform expression. J Clin Invest. 2002; 109(5):629-639.

6. Hu P, Zhang D, Swenson L, Chakrabarti G, Abel ED, Litwin SE. Minimally invasive aortic banding in mice: effects of altered cardiomyocyte insulin signaling during pressure overload. Am J Physiol Heart Circ Physiol. 2003;285(3):H1261-H1269.

7. Shioi $\mathrm{T}$, et al. The conserved phosphoinositide 3-kinase pathway determines heart size in mice. EMBO J. 2000;19(11):2537-2548.

8. McMullen JR, et al. Phosphoinositide 3-kinase $(\mathrm{p} 110 \alpha)$ plays a critical role for the induction of physiological, but not pathological, cardiac hypertrophy. Proc Natl Acad Sci U S A. 2003;100(21):12355-12360.

9. DeBosch B, et al. Akt1 is required for physiological cardiac growth. Circulation. 2006;113(17):2097-2104.

10. Samuelsson AM, et al. Hyperinsulinemia: effect on cardiac mass/function, angiotensin II receptor expression, and insulin signaling pathways. Am J Physiol Heart Circ Physiol. 2006;291(2):H787-H796.

11. Condorelli $G$, et al. Akt induces enhanced myocardial contractility and cell size in vivo in transgenic mice. Proc Natl Acad Sci U S A. 2002;99(19):12333-12338.

12. Matsui $\mathrm{T}$, et al. Phenotypic spectrum caused by transgenic overexpression of activated Akt in the heart. J Biol Chem. 2002;277(25):22896-22901.

\section{Acknowledgments}

We thank Morris J. Birnbaum for Akt1-deficient mice. This work was supported by a Grant-in-Aid for Scientific Research from the Ministry of Education, Science, Sports, and Culture and Health and Labor Sciences research grants (to I. Komuro); a Grant-in-Aid for Scientific Research from the Ministry of Education, Culture, Sports, Science and Technology of Japan; and grants from the Suzuken Memorial Foundation; the Japan Diabetes Foundation; the Ichiro Kanehara Foundation; the Tokyo Biochemical Research Foundation; the Takeda Science Foundation; the Cell Science Research Foundation; the Japan Foundation of Applied Enzymology; and the Astellas Foundation for Research on Metabolic Disorders (to T. Minamino).

Received for publication June 5, 2009, and accepted in revised form February 10, 2010.

Address correspondence to: Issei Komuro, Department of Cardiovascular Science and Medicine, Chiba University Graduate School of Medicine, 1-8-1 Inohana, Chuo-ku, Chiba 260-8670, Japan. Phone: 81.43.226.2097; Fax: 81.43.226.2557; E-mail: komuro-tky@umin.ac.jp.
13. Shioi $\mathrm{T}$, et al. Akt/protein kinase B promotes organ growth in transgenic mice. Mol Cell Biol. 2002; 22(8):2799-2809.

14. Shiojima I, et al. Disruption of coordinated cardiac hypertrophy and angiogenesis contributes to the transition to heart failure. J Clin Invest. 2005; 115(8):2108-2118.

15. Sano M, et al. p53-induced inhibition of Hif-1 causes cardiac dysfunction during pressure overload. Nature. 2007;446(7134):444-448.

16. Gerstein HC, et al. Effects of intensive glucose lowering in type 2 diabetes. $N$ Engl J Med. 2008; $358(24): 2545-2559$

17. Cho $\mathrm{CH}$, et al. Long-term and sustained COMPAng1 induces long-lasting vascular enlargement and enhanced blood flow. Circ Res. 2005;97(1):86-94

18. Abel ED, et al. Cardiac hypertrophy with preserved contractile function after selective deletion of GLUT4 from the heart. JClin Invest. 1999;104(12):1703-1714.

19. Bruning JC, et al. A muscle-specific insulin receptor knockout exhibits features of the metabolic syndrome of NIDDM without altering glucose tolerance. Mol Cell. 1998;2(5):559-569.

20. Zou Y, et al. Mechanical stress activates angioten$\sin$ II type 1 receptor without the involvement of angiotensin II. Nat Cell Biol. 2004;6(6):499-506.

21. Witteles RM, Fowler MB. Insulin-resistant cardiomyopathy clinical evidence, mechanisms, and treatment options. J Am Coll Cardiol. 2008;51(2):93-102.

22. Sharma N, Okere IC, Duda MK, Chess DJ, O'Shea KM, Stanley WC. Potential impact of carbohydrate and fat intake on pathological left ventricular hypertrophy. Cardiovasc Res. 2007;73(2):257-268.

23. Swan JW, et al. Insulin resistance in chronic heart failure: relation to severity and etiology of heart failure. J Am Coll Cardiol. 1997;30(2):527-532.

24. Ingelsson E, Sundstrom J, Arnlov J, Zethelius B, Lind $\mathrm{L}$. Insulin resistance and risk of congestive heart failure. JAMA. 2005;294(3):334-341.

25. Arnlov J, et al. Several factors associated with the insulin resistance syndrome are predictors of left ventricular systolic dysfunction in a male population after 20 years of follow-up. Am Heart J. 2001; 142(4):720-724.

26. Hasegawa S, Kusuoka H, Maruyama K, Nishimura $\mathrm{T}$, Hori M, Hatazawa J. Myocardial positron emission computed tomographic images obtained with fluorine-18 fluoro-2-deoxyglucose predict the response of idiopathic dilated cardiomyopathy patients to beta-blockers. J Am Coll Cardiol. 2004; 43(2):224-233.

27. Garcia-Puig J, Ruilope LM, Luque M, Fernandez J, Ortega R, Dal-Re R. Glucose metabolism in patients with essential hypertension. Am J Med. 2006; 119(4):318-326.

28. Karason K, Sjostrom L, Wallentin I, Peltonen M. Impact of blood pressure and insulin on the relationship between body fat and left ventricular structure. Eur Heart J. 2003;24(16):1500-1505.

29 . Brutsaert DL. Cardiac endothelial-myocardial signaling: its role in cardiac growth, contractile performance, and rhythmicity. Physiol Rev. 2003;83(1):59-115.

30. Neubauer $S$. The failing heart--an engine out of fuel. NEngl J Med. 2007;356(11):1140-1151.

31. Christe ME, Rodgers RL. Altered glucose and fatty acid oxidation in hearts of the spontaneously hypertensive rat. J Mol Cell Cardiol. 1994;26(10):1371-1375.

32. Barger PM, Kelly DP. Fatty acid utilization in the hypertrophied and failing heart: molecular regulatory mechanisms. Am J Med Sci. 1999;318(1):36-42.

33. Davila-Roman VG, et al. Altered myocardial fatty acid and glucose metabolism in idiopathic dilated cardiomyopathy. J Am Coll Cardiol. 2002;40(2):271-277.

34. Yazaki Y, et al. Assessment of myocardial fatty acid metabolic abnormalities in patients with idiopathic dilated cardiomyopathy using 123I BMIPP SPECT: correlation with clinicopathological findings and clinical course. Heart. 1999;81(2):153-159.

35. Cho H, Thorvaldsen JL, Chu Q, Feng F, Birnbaum MJ. Akt1/PKBalpha is required for normal growth but dispensable for maintenance of glucose homeostasis in mice.J Biol Chem. 2001;276(42):38349-38352. 